\title{
Quoi de neuf dans l'histoire du travail ?
}

What's new in the work history?

\section{Florent Le Bot}

\section{OpenEdition}

\section{Journals}

Édition électronique

URL : https://journals.openedition.org/artefact/6836

DOI : 10.4000/artefact.6836

ISSN : 2606-9245

\section{Éditeur :}

Association Artefact. Techniques histoire et sciences humaines, Presses universitaires de Strasbourg

\section{Édition imprimée}

Date de publication : 7 janvier 2021

Pagination : 271-280

ISBN : 978-2-8107-0706-5

ISSN : 2273-0753

Référence électronique

Florent Le Bot, "Quoi de neuf dans l'histoire du travail ? », Artefact [En ligne], 13 | 2020, mis en ligne le 23 décembre 2020, consulté le 28 juin 2022. URL : http://journals.openedition.org/artefact/6836 ; DOI : https://doi.org/10.4000/artefact.6836

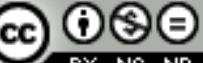

Artefact, Techniques, histoire et sciences humaines est mise à disposition selon les termes de la Licence Creative Commons Attribution - Pas d'Utilisation Commerciale - Pas de Modification 4.0 International. 


\section{Quoi de neuf dans l'histoire du travail ?}

\section{Florent Le Bot}

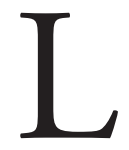

a nouvelle question d'histoire contemporaine posée dans le cadre des concours de l'enseignement (agrégation externe d'histoire, de nité de faire le point sur un objet - le travail - qui a connu un investissement multidisciplinaire conséquent durant les dernières décennies, que ce soit en histoire (économique, sociale, des techniques, culturelle et politique) ou dans une sociologie inscrite en perspective historique et dans d'autres champs disciplinaires. Cette question reflète un dynamisme épistémologique qu'il n'est pas possible de délimiter entièrement en quelques paragraphes. Nous nous contenterons d'esquisser ce qui nous semble en être les lignes de force, en insistant sur l'inscription des articles du dossier dans ces thématiques.

\section{La « révolution industrielle » n'a pas eu lieu}

L'industrialisation ne s'est pas produite partout au même rythme, selon les mêmes temporalités et selon une trajectoire univoque.

Lorsque nous prenons la question en 1830, s'observent un peu partout en Europe de l'Ouest des concentrations manufacturières et minières, un développement des réseaux de transport, qui marquent l'esprit des observateurs

95 Florent Le Bot, «Quoi de neuf dans l'histoire du travail ? », Artefact, 13, 2020, p. 271280. 
(par exemple, Alexis de Tocqueville visitant Manchester en 1835). Pour autant ces foyers ne forment pas l'essentiel du tissu productif du continent ; tant s'en faut. Le travail a d'abord lieu dans les champs et c'est en relation avec ce labeur que se décline toute une pluriactivité. Les lieux du travail ne sont pas pour l'essentiel des "palais de l'industrie " mais bien plus des ateliers et des boutiques, de petites fabriques, de minuscules échoppes, parfois en continuité avec l'espace domestique. Dominent les organisations flexibles où s'entremêlent donneurs d'ordres et apporteurs de travail (à travers le tâcheronnat ou le louage d'ouvrages) ${ }^{1}$, émergences urbaines et ancrages ruraux. Les frontières entre lieux et temps du travail sont elles-mêmes floues et mouvantes. Mais le rythme agraire journalier et saisonnier continue durablement à marquer le tempo des autres productions. Dans les usines elles-mêmes, le travail, l'organisation des tâches et de l'espace, ne se déclinent pas - de manière anachronique - selon les vues du fordisme et du fayolisme du premier $\mathrm{xx}^{\mathrm{e}}$ siècle, mais à travers une composition hétérogène qui fait, par exemple, que le propriétaire des murs n'est pas forcément celui qui emploie la totalité de la main-d'œuvre qui s’y active.

Il n'en est pas moins vrai, qu'un récit s'est construit progressivement autour de l'idée de progrès par l'industrie, les sciences et les techniques. Ce dernier affecte durablement une épistémologie (en histoire, en économie, en sociologie) cherchant à toute fin à définir les origines de la " révolution industrielle ", les phases d'industrialisation, les pays " pionniers " du nordouest européen et ceux en retard, du sud et de l'est. Cette idée trouve sa pleine expression dans le modèle dessiné durant les années 1950 par l'économiste Walt W. Rostow des stades de la croissance (une réponse à Marx, en quelque sorte), imposant l'idée qu'il y aurait une seule et unique voie dans l'industrialisation avec ses étapes obligées, dont le fameux take-off (" le décollage »), que chaque nation emprunterait avec plus ou moins de retard : l'Angleterre aurait engagé le processus, suivie par la Belgique et par la France, rattrapée puis dépassée par l'Allemagne et les États-Unis dès avant la Grande guerre. Ce récit, ensuite décliné à l'échelle de la planète, sert à expliquer au temps de la Guerre froide et des dites "Trente glorieuses ", les retards de développement des pays du "tiers-monde ", ainsi que la meilleure "efficacité " du modèle capitaliste sur le bloc communiste $^{2}$. Le corollaire de cette histoire serait la "supériorité " postulée 
de la grande entreprise intégrée sur toutes les autres configurations entrepreneuriales. Ce paradigme prenant l'allure d'un dogme, s'exprime particulièrement à travers la business history déclinée par l'influent historien Alfred D. Chandler. La crise économique et industrielle ouverte par les années 1970 contribue à mettre à bas ce "beau récit " : les grandes entreprises s'effondrent massivement (à quelques exceptions près) alors que le tissu productif de PME résiste voire se développe, comme les districts industriels de la troisième Italie. Il y avait donc d'autres organisations productives à considérer, d'autres manières de travailler et de produire et d'autres chemins historiques à prendre en considération...

\section{Décentrer le regard}

La notion de " révolution industrielle " a été soigneusement écartée, et à juste titre, par les rédacteurs de la question de concours ; sauf lorsqu'il s'agit d'évoquer son traitement en classe de quatrième - mais entre guillemets, comme elle y figure dans les programmes de l'enseignement secondaire. Cette notion pour être désuète (même si elle est accrochée à un fonds culturel commun) a toutefois marqué la recherche autour de chronologies différenciées d'industrialisation et d'approches territoriales comparées, dans un jeu de décentrement du regard. Les configurations productives, techniques, sociales et spatiales sont de fait extrêmement variées et cohabitent plutôt qu'elles ne se succèdent. François Jarrige rappelle que si le charbon et la force motrice de la vapeur sont les emblèmes de la " révolution industrielle ", les sources d'énergie plus anciennes (les bras, les chevaux et autres bêtes de somme, tout comme le bois et l'hydraulique), loin de disparaître, voient leur place s'accroître au cours du XIX siècle en Europe et au-delà, notamment aux États-Unis. Un manège à chevaux est parfois plus efficace qu'un moteur à vapeur selon le contexte ou le terrain. Des historiens d'envergure, tel Serge Benoit, ont par une pratique du terrain (la Bourgogne et la basse vallée de l'Iton en Normandie, chez ce dernier) nourrie d'archéologie industrielle, contribué à sortir l'histoire des énergies, et donc des processus d'industrialisation, des préjugés légués par de vastes fresques émerveillées par la « modernité ${ }^{3}$ ".

3. Benoit, 2020. 
La comparaison entre Belgique, espaces germaniques, Espagne, France, Grande-Bretagne et Italie, permet de dégager du multiple et de la diversité. Mais la comparaison nous pousse également à resserrer la focale à l'échelle des régions. La Catalogne et le pays basque en Espagne (après l'échec - el fracaso - d'une première industrialisation précoce en Andalousie $)^{4}$, le Piémont en Italie, s’industrialisent plus vite que le reste de leurs péninsules. La comparaison se poursuit à l'échelle des territoires. Jean-Marc Olivier a ainsi caractérisé pour le $\mathrm{XIX}^{\mathrm{e}}$ siècle un espace transfrontalier entre Jura morézien et cantons suisses 5 . Les paysans pluriactifs du haut Jura réussissent une " industrialisation douce " fondée sur l'énergie hydraulique et le travail à domicile, en lien avec des entreprises horlogères suisses, elles-mêmes profondément ancrées dans le monde des campagnes. L'observation depuis les territoires, de la campagne et de la ville, à travers les connections, les circulations, les échanges, les concurrences, parfois l'ignorance mutuelle (également porteuse de sens), permet de sortir des approches déterministes et téléologiques.

\section{À bras-le-corps}

La comparaison nous conduit également à revoir les situations à partir des pratiques, des gestes et des techniques, directement dans l'atelier, la fabrique, la boutique ou au domicile, en se fondant sur l'expérience des acteurs et au prisme des différentes traces que ceux-ci ont laissées et qui sont autant de sources pour l'historien. Si le métier de mineur de charbon, et donc le geste technique minier (la combinaison de la posture et du mouvement du corps appliquée à un outil ou à une machine pour extraire le charbon et le remonter à la surface) n'est plus exercé ni enseigné, la mémoire de ce geste est conservée par les anciens mineurs, par des musées, mais aussi dans les archives des mines ${ }^{6}$.

Le travail marque son empreinte sur les corps et les mets à l'épreuve. La pénibilité au travail est l'un de ces facteurs qui soudent les communautés de production et les métiers. Nombreux sont ceux, des réformateurs sociaux aux pouvoirs publics, qui se préoccupent de la condition ouvrière, pour

\footnotetext{
4. Nadal, 1975.

5. Olivier, 2004.

6. Bouillon, 2017.
} 
surveiller, discipliner, moraliser et assainir les « classes laborieuses, classes dangereuses " mais aussi pour en améliorer le sort. Il n'est donc pas étonnant que les enquêtes ouvrières se multiplient à travers toute l'Europe ${ }^{7}$ : dans les industries du coton, de la laine et de la soie en France avec Louis René Villermé, dans les années 1830 ; dans les cités minières du Borinage belge ; dans les usines Fiat de Mirafiori à Turin dans les années 1960.

L'article de François Jarrige, "La question des machines, le travail et les savoirs au XIX ${ }^{e}$ siècle ", déplace les enjeux de la mécanisation, de la croissance productive vers ce que la machine fait aux hommes et à leur travail : comment ceux-ci se l'approprient-ils? Quel rêve d'opulence formulent-ils ? Quelles craintes et quels cauchemars également? La machine est ainsi cette «tueuse de bras » qui réduit les corps en " automates animés ${ }^{8}$ » et qui contraste avec l'utopie technicienne de maîtrise du monde porté notamment par le groupe émergent des ingénieurs ${ }^{9}$. En 1930, l'économiste Jacques Duboin en vient même à prophétiser une ère d'abondance et d'insouciance grâce à l'emploi extensif des machines ${ }^{10}$. En réalité, ce que nous montre François Jarrige, c'est que dans le processus de mécanisation rien n'est univoque : si les grandes machines renforcent sans nul doute la déqualification (et la concentration) de la main-d'œuvre dans des usines, d'autres, comme la machine à coudre provoquent un mouvement inverse en relançant le travail à domicile. Encore que celle-ci contribue à la définition/redéfinition du partage et de la hiérarchie des tâches entre les hommes et les femmes, de la pérennisation des inégalités à une certaine relégation féminine dans la sphère domestique : les techniques concourent à ordonner le social et particulièrement les relations sexuées ${ }^{11}$. Dans l'industrie porcelainière du second $\mathrm{XIX}^{\mathrm{e}}$ siècle, les nouvelles techniques d'impression induisent des bouleversements tant au niveau de la décoration de la vaisselle et des vases que du point de vue de la main-d'œuvre. Ces procédés permettent une standardisation des objets décorés, un accroissement de la productivité des ateliers et une réduction des coûts : le travail long et minutieux du peintre en porcelaine est remplacé par celui plus fruste des ouvrières décalqueuses. Cette déqualification du travail s'accompagne d'une féminisation

7. Geerkens, Hatzfeld et al., 2019.

8. Jarrige, 2009.

9. Voir l'article de Florent Le Bot et Alain P. Michel dans ce numéro.

10. Dard, 2015.

11. Chabaud-Rychter et Gardey, 2002 ; Perrot, 2006 ; Edgerton, 2013. 
de l'emploi et d'une réduction des salaires ${ }^{12}$. Penser ce que le masculin et le féminin font au travail (et inversement), ainsi qu'aux hiérarchies sociales, aux rémunérations, aux conflictualités, aux représentations, etc. conduit à rétablir le tableau social dans son intégralité.

Larticle de Cédric Perrin sur « ce que l'industrialisation a fait aux artisans d'Europe occidentale » questionne le travail à l'échelle des plus petites entreprises. Le travail familial y était une réalité mais occasionnelle et ponctuelle, relevant de la logique du coup de main, pas toujours rémunéré. Des exceptions sont à relever dans l'alimentation ou dans certaines situations particulières, dont celle des migrants du bâtiment ${ }^{13}$. Le désencastrement du travail de l'univers familial est un phénomène qui reste à interroger. Travailler dans les mines ou les manufactures du XIX ${ }^{\mathrm{e}}$ siècle c'est souvent travailler en famille ; le donneur d'ordre, mineur ou ouvrier lui-même, est souvent le mari et le père. Mais l'intérêt principal de cet article est de nous rappeler ce qui pourrait paraître comme une évidence, mais qui ne l'est pas aux regards de l'historiographie ancienne et des déplorations intéressées : les artisans n'ont pas disparu avec la "révolution industrielle ", même si un secteur comme le textile fut durement frappé. Mieux, de nouveaux métiers, de nouveaux artisans sont apparus dans les services (comme les garagistes, les taxis, les photographes notamment). Cédric Perrin rappelle opportunément que les artisans ne sont pas des "ouvriers sans machines ». Ceux-ci en retirent d'ailleurs une certaine fierté, puisqu'ils les arborent à l'en-tête de leurs courriers.

L'organisation professionnelle, syndicale et politique des mondes du travail est abordée dans les trois textes : que les syndicats discutent des conséquences de la mécanisation chez Jarrige ; que les artisans s'organisent collectivement en Allemagne et en France dès la fin du XIX ${ }^{e}$ siècle puis à l'échelle européenne dans les années 1930, pour faire valoir leurs intérêts collectifs, souvent dans un esprit corporatiste ; ou qu'enfin, dans le texte de Stéphane Lembré, les syndicats contribuent à la formation et à l'information des ouvriers à travers l'envoi de délégations en visite dans les expositions universelles (depuis celle de Crystal Palace à Londres en 1851) et dans les musées, qui sont " la partie la plus visible d'une pédagogie par l'observation et la démonstration, dont le rôle aux côtés de l'apprentissage par la pratique est probablement sous-estimé ».

12. Le Bot, 2013.

13. Martini, 2016. 
Les accidents liés aux mésusages des machines notamment poussent à l'institution d'écoles et de centres de formation : "domestiquer » la machine s'apprend. Mais Stéphane Lembré, dans son article sur « l'industrialisation et la formation au travail ", insiste sur la nécessité de découpler dynamique d'industrialisation et développement des modes et des systèmes de formation. Si de nombreuses institutions de formation destinées aux ouvriers émergent effectivement dans des pays d'Europe occidentale engagés dans une phase historique d'industrialisation, elles ne marquent aucunement la naissance de la formation au travail. En revanche, l'industrialisation vient déplacer les qualifications requises et transforme les modes de transmission. Toutefois, le chemin est tellement peu univoque que, dans les années 1930 encore, se former pour exercer un métier n'a aucune espèce d'évidence, pas plus que n'existait au niveau international, une définition claire et partagée sur ce que pouvaient signifier précisément l'apprentissage et l'enseignement technique et professionnel.

L'enjeu des représentations traverse les trois textes proposés dans ce dossier. Pour Stéphane Lembré, c'est à travers le thème prégnant de " la crise de l'apprentissage " que se manifeste appels à la réforme ou à la contre-réforme : la crise se réfère à "l'âge d'or " d'un monde des corporations où le métier se serait transmis de manière stable de maître à élève et dans lequel la production n'aurait pas eu à souffrir de pénurie de main-d'œuvre. Comme tout "âge d'or ", celui-ci est hautement improbable. L'article éclaire la pluralité des facteurs de transformation des modes de formation. Ce faisant, il ne nie pas la réalité de causes techniques dans la remise en cause de l'apprentissage " traditionnel ", érigeant le lieu de travail en espace évident de la transmission du métier, mais il apprécie de manière plus fine, la place exacte de ces facteurs techniques et les circonstances dans lesquels ils influencent des décisions de création, de réforme voire d'interruption de dispositifs de formation.

\section{Guerres et crises}

Il reste un enjeu abordé dans ces trois articles : ce que la politique et les institutions font au travail. Sans traiter de toutes les dimensions ouvertes par cette clé (par exemple, les enjeux autour des processus d'unification nationale en Allemagne ou en Italie), l'importance des crises et des guerres qui 
s'entremêlent à cette histoire, doit être rappelée. Les crises sont à la fois des révélateurs, des accélérateurs, lorsqu'elles n’interrompent pas des processus. À l'été 1830, nous apprend Jarrige, les Bruxellois qui se rendent en cortège à l'hôtel de ville, crient "Vive Napoléon ! À bas Guillaume ! Brisons les machines! ». En 1848, signale Lembré, une commission d'enquête sur l'industrie linière préconise, parmi différentes mesures pour faire face aux difficultés, d'instituer des écoles d'apprentissage de métiers. Il faudrait évoquer les effets de la guerre franco-prussienne sur le système d'enseignement et de formation français, ou comme Jarrige le fait, évoquer la grande dépression des années 1870-1890.

Mais deux séquences sont marquantes et les trois auteurs s'y réfèrent. La mobilisation massive de 1914-1918 conduit à l'adoption de technologies plus productives et à la diffusion des techniques de rationalisation de la production (Jarrige et Lembré). L'attention portée à l'apprentissage et à l'enseignement technique et professionnel est relancée : certaines entreprises possèdent même leurs propres écoles d'apprentissage (Lembré). C'est à la suite de la guerre que le besoin d'organisation de profession se fait pressant chez les artisans (Perrin).

Dans ce contexte, l'État est un acteur de plus en plus présent.

Dans les années 1920-1930, les régimes autoritaires s'intéressent beaucoup à l'enseignement technique, autant sous l'angle de la moralisation de la classe populaire que dans le cadre de conceptions corporatistes et dirigistes de l'économie. En Espagne, Primo de Rivera (1923-1930) mobilise l'enseignement industriel et la formation professionnelle dans un projet de modernisation économique compatible avec le conservatisme social. Le Troisième Reich combine également formation pratique et endoctrinement politique. La crise des années 1930 et la montée du chômage de masse font rebondir les débats autour de l'industrialisation et notamment de la mécanisation destructrice d'emploi (même si la machine déplace plus qu'elle ne remplace l'homme - y compris de nos jours avec les machines informatiques ${ }^{14}$ ), autour de la formation et de son inadéquation aux besoins, autour de l'artisanat et de son supposé « âge d'or ». Ce thème est repris par les dictatures réactionnaires de Salazar au Portugal et de Pétain en France,

14. Casilli, 2019. 
mais la valorisation du " petit artisan " contre le « gros capitaliste » est un thème qui traverse toute une part de la littérature occidentale à l'époque.

En 1937, Jean Giono confrontant le sort de son père cordonnier à celui de la grande firme de chaussures Bata implantée un peu partout en Europe et sur les autres continents, déplore :

Louvrier de Bata met à peine une demi-heure [à coudre une trépointe]. Il y est plus habile que mon père mais il ne sait faire que ça. Il ne sait pas monter tout le soulier. [...] L'ouvrier ne peut quitter sa chaise chez Bata. S'il s'en allait de là il ne pourrait pas vivre. Il n'a plus un métier qui le fait vivre n’importe où. [...] Il est prisonnier et sa famille est prisonnière. Et, si on lui donne quinze jours de congés payés par an, je dis qu'à côté des grandes vacances perpétuelles de mon père ce qu'on appelle ici progrès n'est qu'une sérieuse régression ${ }^{15}$.

La littérature est partie prenante des représentations des mondes du travail, que ce soit sous une forme documentaire ou, comme ici, inscrite dans une lecture orientée des réalités. Il faudra sans doute du temps avant que ne s'estompe la fiction d'une "révolution industrielle ".

\section{Bibliographie}

Benort Serge, D'eau et de feu : forges et énergie hydraulique, XVIII'-XX' siècle. Une histoire singulière de l'industrialisation française, PUR, Rennes, 2020.

Bouillon Didier, Guillerme André, Mille Martine et Piernas Gersende (dir.), Gestes techniques, techniques du geste, préface Denis Woronoff, PU du Septentrion, Villeneuve d'Ascq, 2017.

Casilli, Antonio A., En attendant les robots. Enquête sur le travail du clic, Seuil, Paris, 2019.

Chabaud-Rycher Danielle et Gardey Delphine (dir.), L'Engendrement des choses, Archives Contemporaines, Paris, 2002.

DARD Olivier, "Sortir de la crise par la technique et la science ? Les réponses de l'abondancisme de Jacques Duboin ", dans Dard Olivier, Didry Claude, Le Bot Florent et Perrin Cédric (dir.), "Les mille peaux du capitalisme ", L'Homme et la société, n ${ }^{\circ}$ 195-196/2015, p. 127-145.

DIDRY Claude, L'Institution du travail. Droit et salariat dans l'histoire, La Dispute, Paris, 2016.

15. Le Bot, 2009. 
EDgerton David, Quoi de neuf? Du rôle des techniques dans l'histoire globale, Seuil, Paris, 2013.

Geerkens Éric, Hatzfeld Nicolas, Lespinet-Moret Isabelle et Vigna Xavier (dir.), Les Enquêtes ouvrières dans l'Europe contemporaine, La Découverte, Paris, 2019.

JaRRige François, Au temps des "tueuses à bras ». Les bris de machine à l'aube de l'ère industrielle, 1780-1860, PUR, Rennes, 2009.

Le Bot Florent, "Dans l'atelier du cordonnier. Guéhenno, Giono, Guilloux, artisans pacifistes », dans Guérin Jean-Yves, PaulHan Jean-Kely et Rioux JeanPierre (dir.), Jean Guéhenno. Guerres et paix, PU du Septentrion, Villeneuve d'Ascq, 2009, p. 105-118.

LE Bot Florent, « La porcelaine de Limoges : décorée ou en blanc? (1840-1940) », dans Lamard Pierre et Stoskopf Nicolas (dir.), Art et industrie, XVII -XXI siècle, A. et J. Picard, Paris, 2013, p. 165-176.

Martini Manuela, Le Bâtiment en famille. Migrations et petite entreprise en banlieue parisienne au $\mathrm{xx}^{\mathrm{e}}$ siècle, CNRS Éditions, Paris, 2016.

Martini Manuela, "Tâcherons ou sous-traitants ? Travailleurs indépendants et entrepreneurs dans la construction en France entre la fin du XIx ${ }^{e}$ siècle et l'entre-deux-guerres ", Revue de synthèse, 140/1-2, 2019, p. 43-84.

Nadal Jordi, El fracaso de la Revolución industrial en España, 1814-1913, Barcelone, Ariel, 1975.

Olivier Jean-Marc, Des clous, des horloges et des lunettes. Les campagnards moréziens en industrie (1780-1914), CTHS, Paris, 2004.

Perrot Michèle, "Le travail des femmes ", Mon histoire des femmes, Seuil, Paris, 2006, p. 143-180.

Pessis Céline, Topçu Sezin, Bonneuil Christophe (dir.), Une autre histoire des Trente Glorieuses. Modernisation, contestations et pollutions dans la France d'aprèsguerre, La Découverte, Paris, 2013.

\section{L'auteur}

Florent Le Bot est enseignant-chercheur en Histoire contemporaine à l'Université d'Évry Paris-Saclay, IDHES (UMR CNRS 8533). Ses recherches portent sur l'histoire économique et industrielle de l'Europe de l'Ouest aux $\mathrm{xIx} \mathrm{e}^{\mathrm{e}}$ et $\mathrm{xx}$ siècles. Il a notamment publié La fabrique réactionnaire. Antisémitisme, corporatisme et spoliations dans le cuir, Paris, Presses de Sciences Po, 2007 ; Histoire économique de Vichy. L'État, les hommes, les entreprises (avec F. Grenard et C. Perrin), Paris, Perrin, 2017 et L'échelle des régulations politiques, XVIII'-XXI siècles. L'histoire et les sciences sociales aux prises avec les normes, les acteurs et les institutions (avec V. Albe et J. Commaille, dir.), Villeneuve-d'Ascq, Presses universitaires du Septentrion, 2019. Contact : florent.lebot@univ-evry.fr 